\title{
Apoyo emocional: conceptualización y actuación de enfermería, una mirada desde la perspectiva del alumno
}

\author{
Emotional support: conceptualization and performance \\ of nursing, a look from the perspective of the learner
}

Mtra. María Dolores Martínez Garduño*, Lic. Vicenta Gómez Martínez*, Lic. Piedad Siles Juárez*

*Profesora de tiempo completo Fac. de Enf. UAEMéx, ${ }^{* \star}$ Lic. en Enfermería, adscrita Centro Médico

Lic. Adolfo López Mateos, Instituto de Salud del Estado de México.

\section{Resumen}

Durante la formación de enfermería se hace hincapié en el apoyo emocional al paciente, destacando su importancia en el cuidado integral, sin embargo se carece de definición clara sobre este concepto y en muchas ocasiones, éste no se brinda. El objetivo fue identificar la conceptualización y cuidado de apoyo emocional que brindan los alumnos de la licenciatura en Enfermería.

Metodologia: El estudio fue descriptivo y transversal. El universo 210 alumnos. Las categorías del instrumento: conocimiento, identificación, planeación y evaluación de intervenciones para brindar apoyo emocional.

Una minoría define apoyo emocional como "Dar confianza, empatía y dar a conocer lo que le va a realizar al paciente".

Resultados: El conocimiento de la patología es prioritario (16\%) las acciones que realizan están encaminadas a favorecer la conversación como medio de disminución de la respuesta emocional (44\%); considera que es necesario ayudar o animar al paciente a expresar sus sentimientos (39\%); considera que se debe platicar con el paciente sobre algo ajeno a su enfermedad para brindar apoyo emocional 16\%; se debe brindar apoyo emocional realizando los procedimientos sin hablarle al paciente es la opinión del .55\%.

Es imprescindible hacer evidente la necesidad de crear una escala que facilite la valoración, y la evaluación de las intervenciones de enfermería con relación a las emociones sentidas y expresadas por los pacientes.

Palabras clave: enfermería, apoyo emocional, alumno. 


\section{Abstract}

During the training of nursing emphasizes the emotional, stressing their importance for the patient care, however there is not clear definition of this concept and many times this support is not provided. The aim was to identify the design and care of emotional support provided by students of the Bachelorship in Nursing. Methodology: the study was descriptive and transversal. The universe was 210 students. The instrumental categories: awareness, identification, planning and evaluation of interventions to provide emotional support.

A minority of the students defined emotional support as: "trust, empathy and awareness of what the patient is going to do". Results:Knowledge about pathology is a priority (16\%). Actions that are undertaken to encourage the conversation as a means of reducing the emotional response (44\%), percentage who believe it is necessary to assist or encourage the patient to express their feelings (39\%), percentage who believed that talking with the patient about something unrelated to his illness to provide emotional support; $16 \%$ and percentage who reported that emotional support should be done by following procedures without talking to the patient $.55 \%$. There is clear need to create a scale to facilitate the assessment and evaluation of nursing interventions in relation to the emotions felt and expressed by the patients.

Keywords: nursing, emotional support, student.

\section{INTRODUCCIÓN}

Conocer a un paciente significa que la enfermera evita suposiciones, se centra en él y pone en marcha una relación de cuidados que le proporcione información e indicios que facilitan el pensamiento crítico y los juicios clínicos que va a realizar.

Cuando se aplica a la persona, el concepto de cuidado, se debe tomar en cuenta que este deberá ser holístico, resaltando el hecho de que las enfermeras deben conservar en su mente a la persona de manera integral, y esforzarse por comprender la zona de preocupación del paciente que guarda relación con sus esferas biológica, psicológica, social y cultural.

El profesional de enfermería, como ente proveedor de servicios de salud, realiza un acto personal, ético y solidario en el proceso salud-enfermedad ${ }^{1}$, en los pacientes que precisan de una atención adecuada.

Para brindar ese apoyo que requieren los pacientes, el alumno debe contar con un cuerpo de conocimientos científicos, tomando en cuenta que al individuo se le contempla en sus tres esferas; biológica, social y psicológica. Esta última abarca el apoyo emocional que "comprende la empatía, el cuidado, el amor y la confianza que una persona ofrece a otra"2, por consiguiente la enfermera debe tener los conocimientos necesarios y la sensibilidad para poder atender y brindar esta necesidad, que el individuo demanda día con día. Respecto al apoyo emocional que el alumno de la Licenciatura en Enfermería debe brindar al individuo sano o enfermo, se ha observado que no se

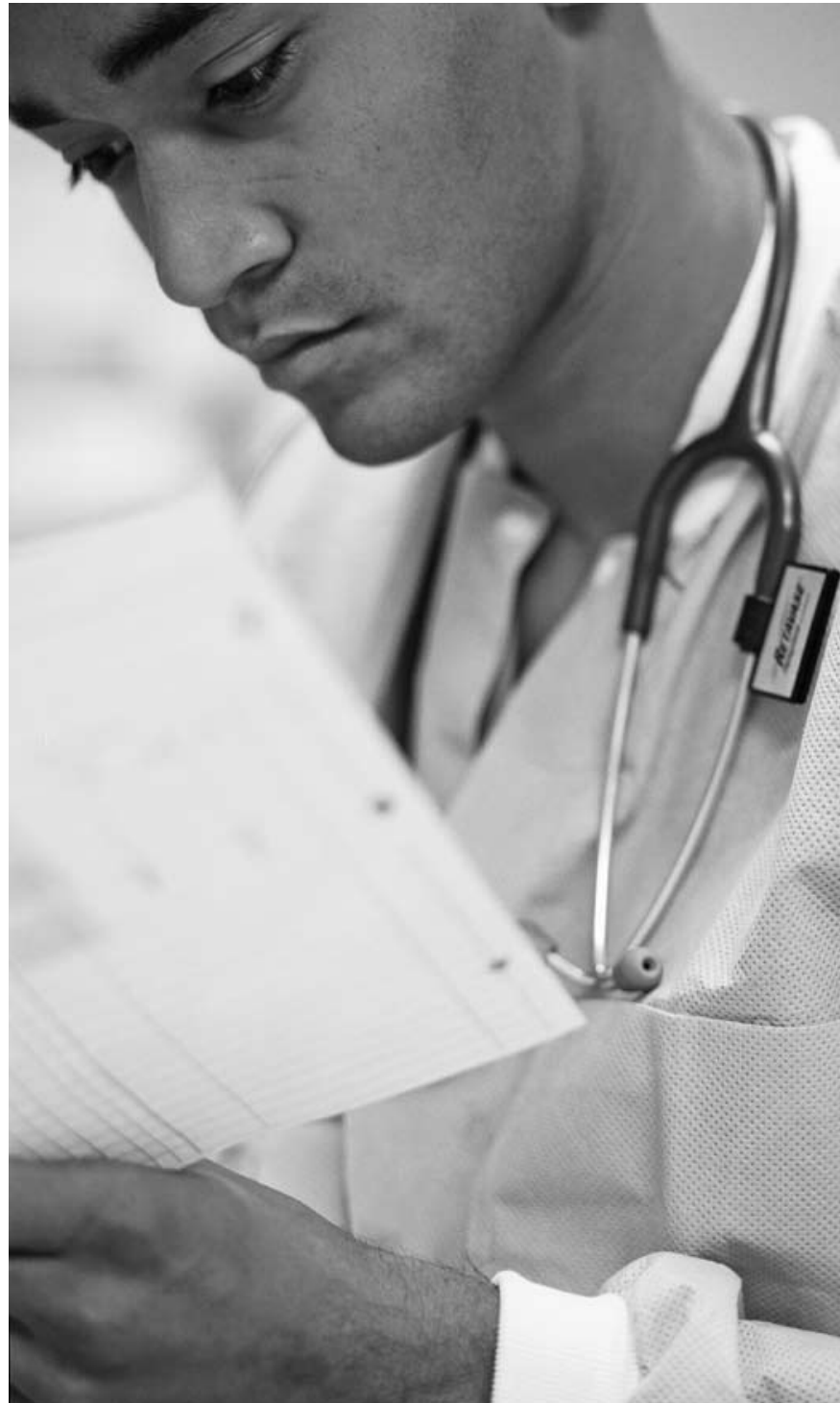


atiende completamente esta necesidad, puesto que necesita tener una adecuada interacción con el paciente, de igual forma debe brindar apoyo y atención de acuerdo a la identificación de necesidades. Se debe enfatizar durante la formación de los alumnos que en primer término es imprescindible brindar apoyo emocional, pero además, en la bibliografía este concepto es muy ambiguo.

Debe entenderse entonces que el alumno desarrollará su propio concepto de lo que es apoyo emocional, por lo tanto, durante su formación académica, dentro de la teoría pone como eje principal el apoyo emocional en los cuidados que va a brindar, y es de esperarse que en la práctica lo lleve a cabo, desde su propio enfoque y conceptualización. Cabe mencionar que existen pocos estudios sobre el apoyo emocional que brinda la enfermera, así como las posibles intervenciones que deberá realizar. ${ }^{3}$

El desarrollo del conocimiento de enfermería y sus intervenciones implica en su práctica profesional, el proporcionar apoyo emocional; entendiéndose como apoyo cuando se presta ayuda desinteresadamente, generalmente se da en las personas que necesitan algún tipo de ayuda, de cualquier índole. La palabra emoción deriva del latín emovere, que quiere decir remover, agitar o excitar. Se definirá como un estado efectivo pasajero, pero es el motor que incita a realizar una acción, mueve, conmueve y motiva la conducta. Las emociones son un estado de ánimo, de humor que se les atribuye un comportamiento, ocasionan un aumento en el nivel de actividad de nuestro organismo. ${ }^{4}$

Se puede encontrar entonces que hay una necesidad de apoyo y acciones ajustadas a la situación de vida de un paciente. Estas acciones deben contribuir a aumentar el nivel de apoyo emocional y de influir positivamente en el nivel de satisfacción con la cantidad de apoyo social que recibe el paciente, a su vez, ofrecer una mejor convalecencia, por parte de las personas que se encuentren a su alrededor. ${ }^{5}$

\section{METODOLOGÍA}

\section{El diseño de este estudio fue transversal y descriptivo.}

El universo de estudio fueron alumnos del último semestre de la carrera de la Licenciatura en Enfermería, siendo un total de alumnos de 210. La muestra fue no probabilística y por conveniencia, formada por 180 alumnos. Solo se excluyeron aquellos que no se encontraron presentes el día de la aplicación.
Se estructuró un instrumento, con 18 ítems; después de la aplicación piloto en una población de 30 sujetos con características similares, se eliminaron 5 ítems y se agrego uno, quedando de 14 ítems; 11 ítems de opción múltiple, 3 de complementación y una parte de datos sociodemográficos. Las categorías que contiene el instrumento son: conocimiento sobre apoyo emocional e identificación de los estados emocionales, planeación de las posibles intervenciones, presunción de la necesidad de apoyo emocional, intervención y evaluación de las posibles intervenciones. Se obtuvo un alfa de Cronbach de $.777 \mathrm{El}$ análisis de los resultados se elaboró con una base de datos en el programa SPSS Versión 13, se utilizó como unidad de análisis estadístico frecuencias y porcentaje, los resultados se presentaron en cuadros y/o gráficas. Finalmente se establecieron las conclusiones de la investigación realizada y propusieron sugerencias de la investigación.

\section{RESULTADOS}

Dentro de las definiciones dadas por los alumnos acerca de apoyo emocional el 39\% lo definió como "Dar confianza, empatía y dar a conocer lo que se le va a realizar", $31 \%$ lo define como "Brindar cuidados enfocados a los sentimientos", para el 21\% lo consiste en el "Apoyo que se brinda a las personas en sus tres esferas, para el resto (9\%) es el "Brindar apoyo para que se recupere".

De acuerdo a los estados emocionales que presenta el individuo, el $59 \%$ de los alumnos identifica miedo y/o sorpresa en los pacientes, 34\% identifica tristeza, otro 3\% identifica alegría y el $4 \%$ restante refiere identificar ira y/o disgusto. Para el $46 \%$ de los alumnos, el conocimiento de la patología es prioritario, mientras que el $41 \%$ le interesa conocer el nombre del paciente, otro $9 \%$ refiere que le interesa estar al tanto de los procedimiento que se va a realizar, solo el 3\% de los alumnos encuestados les intereso conocer mas de un dato del paciente, entre estos datos están: nombre y la patología y menos del uno por ciento (.55\%) solo le interesa conocer la edad.

Respecto al tiempo que se brinda el apoyo emocional, el 58\% de los alumnos brindan cuidado emocional de 10 a 20 minutos, el $27 \%$ de 20 a 30 minutos, el $12 \%$ menos de 10 minutos, $2 \%$ realiza hasta mas de 40 minutos y solo $1 \%$ lo realiza de 30040 minutos. Estos datos permiten al alumno realizar planeación de las posibles intervenciones.

Las acciones que el alumno encuestado brinda al paciente son: favorecer la conversación como medio de 
disminución de la respuesta emocional para poder brindar apoyo emocional, esto lo refiere 44\%; 39\% considera que es necesario ayudar o animar al paciente a que exprese sus sentimientos y $16 \%$ considera que se debe platicar con el paciente sobre algo ajeno a su enfermedad para brindar apoyo emocional y el otro $1 \%$ refiere que se debe brindar apoyo emocional realizando los procedimientos sin hablarle al paciente. Uno de los primeros cuidados que brinda el alumno en un $67 \%$ es apoyo emocional, mientras que $30 \%$ el primer cuidado que brindan es la toma de signos vitales, $2 \%$ es la aplicación de medicamentos y solo $1 \%$ el primer cuidado que brinda es curar la herida si es que la tiene.

De los alumnos encuestados el $80 \%$ considera que siempre es indispensable brindar apoyo emocional, mientras que solo el $19 \%$ opina que solo algunas veces debe brindarse apoyo emocional y $1 \%$ refiere que es de poca importancia brindar apoyo emocional.
Con relación a la evaluación de las intervenciones que realiza el alumno para atender los estados emocionales del paciente en un $41 \%$ es por medio de la actitud demostrada por el paciente al personal de enfermería, el 20\% no contesto la pregunta, un 14\% considera que la evaluación es insuficiente debido a la carga de trabajo excesiva del personal de enfermería por ello no se puede brindar un cuidado adecuado, otro 14\% refieren que por medio del estado físico y emocional, $11 \%$ considero que es dependiendo de la enfermedad y del estado del paciente.

\section{DISCUSIÓN}

El término de apoyo emocional es poco discutido, el más comúnmente empleado es el de apoyo social (social support ${ }^{6}$ sin embargo hay puntos de convergencia. De acuerdo a los datos observados, se identificó que más de la mitad de los alumnos encuestados definen al apoyo emocional como lo define Méndez ${ }^{7}$

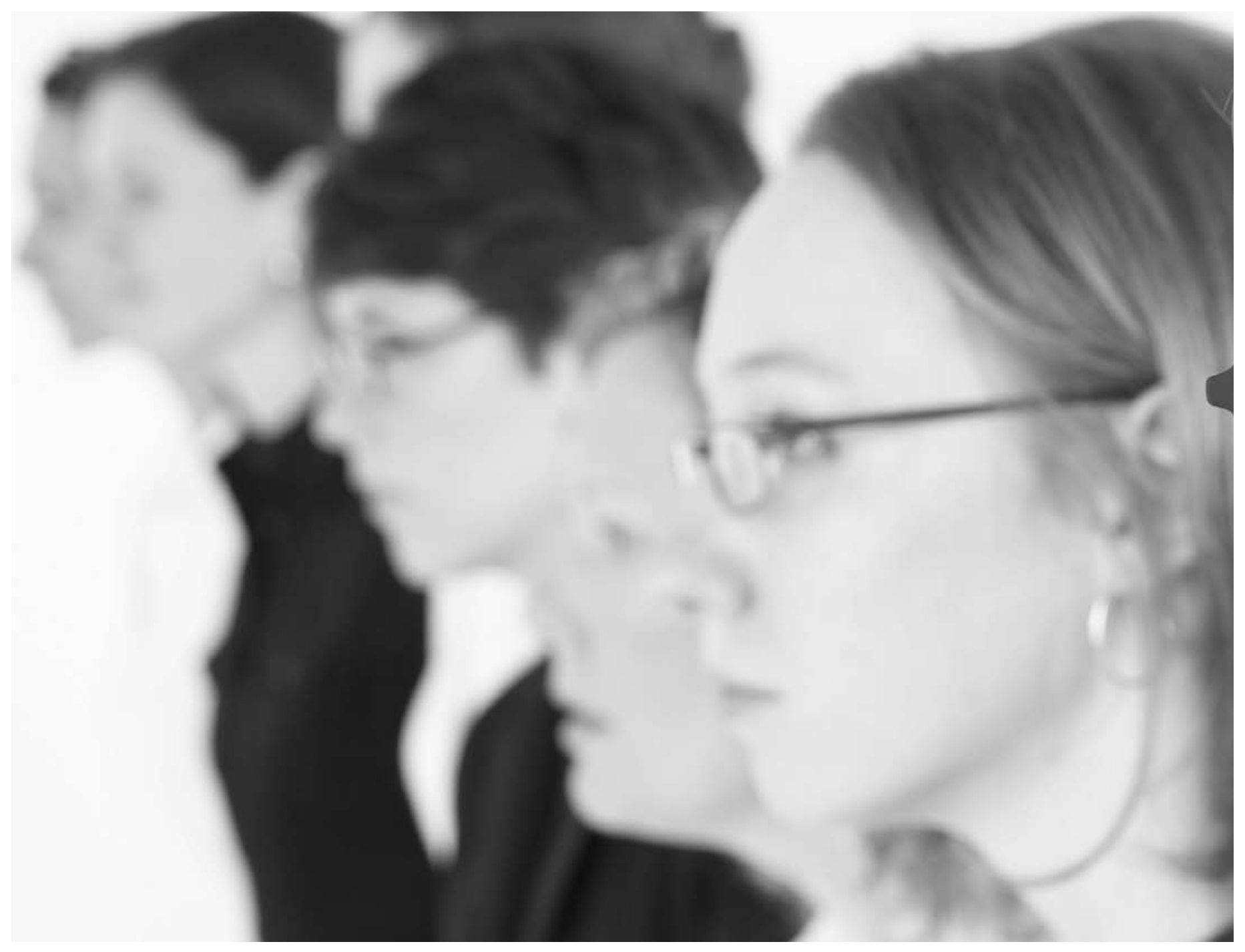


"Brindar empatía, cuidado, amor y confianza al individuo", algunos alumnos consideran que apoyo emocional es: "proporcionarle seguridad, aceptación y ánimo en momentos de tensión al individuo" Por lo que se coincide que estas definiciones pueden ser similares para definir apoyo emocional. ${ }^{\text {h }}$ Mientras que el resto de los alumnos encuestados solo tienen una pequeña noción de lo que es apoyo emocional, ya que consideran que solo es animar al paciente a expresar emociones y ayudarle en la toma de decisiones, definiciones que no se encuentran en la bibliografía.

Como punto importante de esta investigación la mayoría de los alumnos admiten que uno de los primeros cuidados que se debe brindar a los pacientes hospitalizados, es el brindar apoyo emocional, situación que conllevará a brindar un adecuado cuidado de calidad y calidez. Los alumnos consideran importante, dedicar un tiempo al apoyo emocional tal y como lo sugiere Armayones ${ }^{9}$ siendo trascendental que el tiempo que se toma la enfermera para realizar cuidado, escuchar (escuchar con eficacia) vale la pena, tanto por la información obtenida como por lo que refuerza la relación enfermera-paciente.

A los alumnos no se les dan a conocer escalas para evaluar estados emocionales, probablemente porque estas no existan o no se conocen, pero si se han descrito aspectos psicológicos de bienestar que pueden ser atendidos desde la perspectiva de la enfermería psicosocial. ${ }^{10}$

\section{CONCLUSIONES}

Aun es débil la fiabilidad obtenida del instrumento, es necesario repetir su aplicación en una población más amplia y someter el instrumento a una validez de contenido a través de un jueceo de expertos Sin embargo de la población estudiada, se puede afirmar que prevalece una formación donde su enfoque de indagación e intervención se dirige más hacia un pragmatismo, dándole mayor importancia a los denominados datos fríos traducidos a números (signos vitales, datos de laboratorio, cruces en una glucosuria, etc.), restándole importancia al aspecto emocional.

Al no contar con un concepto claro de lo que es el apoyo emocional, las posibles intervenciones se reducidas a los aspecto técnico administrativos, aun cuando existen teorías de enfermería que pudieran ser aplicadas, tal es el caso de Joyce Travelbee quien cuenta con un modelo fundamentado en el aspecto emocional y Joan Riel-Sisca; en un modelo de interaccionismo simbólico. ${ }^{11}$
Finalmente es imprescindible hacer evidente la necesidad de crear una escala que facilite la valoración, y la evaluación de las intervenciones de enfermería con relación a las emociones sentidas y expresadas por los pacientes.

\section{REFERENCIAS BIBLIOGRÁFICAS}

1 Gastman Chris. Schotsman Paul. Nursing Considered as Moral Practice: A Philosophical-Ethical Interpretation of Nursing. Kemmedy Institute of Ethics Journal Vo.8 1 March 1998 pp 43-69

2 Langford C.P.H.; Bowsher J.; Maloney J.P.; Lillis P.P. Social support: a conceptual analysis. Journal of Advanced Nursing. Volume 25 Issue 1, Pages 95-100. Published on line 28 Jun 2008. Disponible en: http://www3.interscience. wiley.com/cgi-bin/fulltext/119155643/PDFSTART

3 Fernández Abascal Enrique, Jiménez Sánchez María del Pilar. Martin Díaz Ma. Dolores. Emoción y motivación La adaptación humana. E. Centro de estudios Ramón Areces.S.A., España 20031176 pp.

4 Fernandez Op. cit.

5 Kurowska K. Koscielna H. Life orientation and social support in patients with colostomy for colorectal cancer WSPOLCZESNA ONKOLOGIA-CONTEMPORARY ONCOLOGY. Volume: 12 Issue: 5 2008. Pages: 228-233

6 Langford Op. cit.

7 Méndez P. Barra E. Apoyo Social Percibido en Adolescentes Infractores de Ley y no Infractores. PSYKHE 2008, Vol.17, No1, 59-64. Disponible en: http://www.scielo.cl/scielo. php?pid=S0718-22282008000100006\&script=sci_arttext

8 Grupo ASANEC de Inmigración Atención de Enfermería en Población de origen extranjero. Biblioteca Lascasas 2006; 2 Disponible en http://www.index-f.com/lascasas/ documentos/lc0104.php

9 Armayones Ruiz M. Técnicas de Apoyo Psicológico y social. Como desarrollar las habilidades adecuadas ante situaciones de emergencia. Publicado por ideas propias Editorial S.L.España 2007 pp.111

10 Novel Martí Gloria, Lluch Canut Ma. Teresa, Miguel López de Vergara Ma. Dolores. Enfermería Psicosocial y Salud mental. Ed. Elsevier España 2005333 pp.

11 Marriner Tomey Ann, Raile Alligod Martha. Modelos y teorías en Enfermería. 6ª . ed. Elsevier España 2007828 pp.

\section{DIRECCIÓN PARA CORRESPONDENCIA}

Mtra. Ma. Dolores Martínez Garduño: dolmaga@hotmail.com 